\title{
Turnpike sets in stochastic manufacturing systems with finite time horizon*
}

\author{
Q. Zhang ${ }^{\dagger}$ and G. Yin
}

\begin{abstract}
This paper is concerned with finite horizon optimal production planning of stochastic manufacturing systems. The main objective is to minimize an expected discounted cost of inventories and/or backlogs. Owing to the finite time horizon formulation, the dynamics of the system are time inhomogeneous. Thus the turnpike sets of the problem are time varying. Systems with random decreasing capacity and constant demand, and systems with random increasing demand and constant capacity are dealt with. We derive explicit solutions, and show that the optimal controls can be written in terms of the turnpike sets under 'traceability' conditions.
\end{abstract}

Keywords. stochastic production planning, finite time horizon, turnpike sets, traceable curves.

${ }^{*}$ This research was supported in part by the National Science Foundation.

†Department of Mathematics, University of Kentucky, Lexington, KY 40506

$\ddagger$ Department of Mathematics, Wayne State University, Detroit, MI 48202 


\section{Introduction}

This work is concerned with finite horizon production planning of stochastic manufacturing systems. Two problems will be considered. In the first problem, a manufacturing system with a machine subject to breakdown and constant product demand rate is considered, and in the second problem, a manufacturing system with constant production capacity and increasing (random jump) product demand rate is dealt with. These two problems are motived by various applications in industry and management sciences. In particular, the following two examples can be thought of as prototype problems.

A coal mine plant example. Consider the quarterly production plans for a local coal mine plant. The plant is expecting a general strike during a quarter which will reduce the production capacity. If the strike does happen and the dispute cannot be resolved during the quarter, what should the production plans be to meet a given fixed demand rate at the minimum cost of inventories and backlogs?

A new car model example. An automobile manufacturer introduces a new car model. The initial market demand for the new model is relative low. It is expected that due to the increasing customer awareness, the demand for the new model will increase. The objective is again to determine the production plans so as to minimize the total inventory and backlog costs.

In these two production planing problems, by taking the difference of the real and planned cumulative goods of the finished ones as the states of the system, the objective is to choose admissible production rates to minimize the inventory/backlog costs over a finite horizon.

Recently, production planning for manufacturing systems has received much attention. Kimemia and Gershwin [8] studied a multi-machine flowshop without internal buffers known also as a no-wait flowshop. By using a quadratic approximation for the 'cost to go', a linear programming approach, which can be implemented in real time, was suggested. Under a dynamic program formulation, numerical solutions were found, which yield the optimality and a suboptimal control policy defined by two threshold levels. Motivated by the work [8], Akella and Kumar [1] formulated a one-machine, one-part problem as a stochastic optimal control problem, in which the part demand was assumed to be a constant, the state of the machine was assumed to be a two-state continuous time Markov chain, and the objective function was a discounted inventory/shortage cost over an infinite time horizon. It was rigorously proved that the optimal control is given by a single threshold inventory level (or turnpike set). More specifically, they showed that whenever the machine is up, one should 
produce at the maximum possible rate if the inventory level is less than the threshold, produce at the demand level if the inventory level is exactly equal to the threshold, and produce nothing at all if the inventory level exceeds the threshold. A similar problem with the discounted cost replaced by a long run average cost was dealt with in Bielecki and Kumar [3]; optimal threshold type of policies were obtained. In both [1] and [3], explicit formulas for the optimal threshold levels were derived. Further study on turnpike sets were carried out by Sethi et al. [9]. The model considered in [1] was generalized to incorporate systems with more than one states and with random demand processes in [9]; it was proved that the turnpike sets exhibit a monotone property with respect to capacity and demand.

In view of the aforementioned developments, the first and foremost important task appears to be the utilization of turnpike sets. By and large, solving the optimal control problems for the manufacturing systems is equivalent to finding the corresponding turnpike sets.

As far as the control of manufacturing systems is concerned, the infinite time horizon problems can be thought of as long term planning problems, whereas the finite horizon problems may be regarded as short term or intermediate term planning problems. Evidently, the finite horizon problems are as interesting and important as their infinite time counterparts.

First, in various applications arisen from industry, many optimal control problems of manufacturing systems are involved with a finite time horizon. For instance, in the coal mine plant example and the new car production model, the time horizons consist of a quarter and a year, respectively. Quite often, owing to a short term goal or an intermediate term objective, a system manager is more interested in finding optimal or suboptimal strategies for a finite duration rather than that of the distant future. Secondly, to some extent, the optimal control of a infinite horizon problem can be obtained as the limit of finite time problems. As illustrated in Example 3.1 of this paper, using a cost functional associated with the time interval $[s, T]$ together with a discount factor $\rho$, the result (in particular, the optimal threshold value) obtained in [1] is recovered when $T \rightarrow \infty$.

Since a large amount of production planning deals with finite time formulation, and since the infinite horizon problems can be considered as limits of the corresponding finite horizon ones, the study of finite horizon problems is not only worthwhile, but also necessary.

We notice that there are some distinct differences between the finite time and the infinite horizon formulations. For an infinite horizon formulation, such as the problems studied in [1] and [3], the dynamics of the systems are essentially homogeneous, and as a consequence, the turnpike sets consist of constants, which completely characterize the optimal control policies. If the system performance 
is evaluated over a finite time horizon (with initial time $s$ which can be changing), the threshold levels are no longer constants, but "time dependent threshold curves". Therefore, the problem becomes much more complicated. Naturally, one expects that the essence of the turnpike sets that is, produce at the maximum speed if the inventory level is below the turnpike, produce nothing if the inventory level is above the turnpike, and produce exactly as the demand if the inventory reaches the turnpike, should still work. Nevertheless, the time inhomogeneous nature makes it very difficult to obtain explicit optimal solutions as in [1] and [3]. In order to fulfill our goal of achieving the optimality, the turnpike sets must be smooth enough and be "traceable" by the trajectory of the system.

With the main objective in mind, by considering relatively simple models, we aim at deriving explicit optimal solutions. To begin with, new definitions of time varying turnpike sets (or threshold functions) are given. To make the trajectory of the system have required tracing or tracking ability, the notion of "traceable curves" is introduced. Loosely, the traceability of the system requires the derivatives of the turnpike sets to be contained in certain domain.

Since the desired optimal solutions are closely related to the turnpike sets or optimal inventory levels, some detailed discussions are made around these topics. A number of properties of traceable curves are exploited and analyzed. They are, in fact interesting on their own right. The notion of traceability is then employed in the subsequent investigation to develop the desired optimal controls. It will be shown that indeed, there exist turnpike sets and the optimal control can be determined explicitly by such inventory levels. For both the problem with random machine capacity and with constant demand, and the problem with constant machine capacity and random demand, the underlying traceable curves are characterized, and explicit solutions of the optimal controls are exhibited. Some examples are also provided. It should be pointed out that the optimal solutions given in this paper are easy to apply and simple to implement, so the advantage of turnpike sets of the infinite horizon problems is preserved. We hope that the results of this paper will provide further insight on the nature of the underlying problem and heuristics for practical applications.

The plan of this paper is as follows. The formulations of the control problems are given next. In $\S 3$, we concentrate on the first problem - a system with constant demand, and in $\S 4$, we treat the second problem - a system with constant machine capacity. For each problem, explicit formula of the optimal control is obtained. To ensure the continuity and the stream line of the paper, proofs of several lemmas and a number of technical complements are provided in a separate section $\S 5$. Finally, some concluding remarks are made in $\S 6$. 


\section{Problem formulation}

Manufacturing systems with constant demand and with constant machine capability will be dealt with in the sequel. The precise formulations of the problems are given below.

\section{Problem 1: Constant product demand}

Let $x_{t} \in R^{1}$ denote the inventory/backlog process (state variable) and $u_{t} \geq 0$ denote the rate of production planning (control variable) of a manufacturing system. The product demand is assumed to be a constant and denoted by $z$. Then,

$$
\dot{x}_{t}=u_{t}-z, x_{s}=x, 0 \leq s \leq t \leq T
$$

where $T$ is a finite horizon.

Let $\mathcal{M}=\left\{\alpha_{1}, \alpha_{2}\right\}\left(\alpha_{1}>\alpha_{2} \geq 0\right)$ denote the set of machine states and let $\alpha(t) \in \mathcal{M}$ denote the machine capacity process in the manufacturing system. If $\alpha(t)=\alpha_{1}$, it means the machine is in a good condition with capacity $\alpha_{1}$. If $\alpha(t)=\alpha_{2}$, the machine (or part of the machine) breaks down with a remaining capacity $\alpha_{2}$. We always assume that $\alpha_{1}>z>\alpha_{2}$, i.e., the demand can be satisfied if the machine is in a good condition and cannot be satisfied if the machine (or part of the machine) breaks down.

Let the cost function $J(s, x, \alpha, u$.) with $\alpha(s)=\alpha \in \mathcal{M}$ be defined by

$$
J(s, x, \alpha, u .)=E \int_{s}^{T} e^{-\rho t} h\left(x_{t}\right) d t
$$

where $\rho \geq 0$ is the discount factor, which is allowed to be zero, since we are now considering a finite horizon problem. The problem is to find a production plan $0 \leq u_{t} \leq \alpha(t)$ as a function of the past $\alpha(\cdot)$ that minimizes $J(s, x, \alpha, u$.).

\section{Problem 2: Constant machine capacity}

The second problem is a slight variation of the first one. In this problem, the system having a constant machine capacity $\alpha_{0}>0$ with a random demand rate $z_{t}$, is described by the following equation

$$
\dot{x}_{t}=u_{t}-z_{t}, x_{s}=x, 0 \leq s \leq t \leq T<\infty
$$

with the production constraints $0 \leq u_{t} \leq \alpha_{0}$.

Let $\mathcal{Z}=\left\{z_{1}, z_{2}\right\}$ denote the set of demand rates with $0<z_{1}<\alpha_{0}<z_{2}$. 
The corresponding cost function $J(s, x, z, u$. $)$ with $z_{s}=z \in \mathcal{Z}$ is defined by

$$
J(s, x, z, u .)=E \int_{s}^{T} e^{-\rho t} h\left(x_{t}\right) d t
$$

The problem is again to find a production plan $0 \leq u_{t} \leq \alpha_{0}$ as a function of the past $z_{t}$, that minimizes $J(s, x, z, u$. $)$.

The detailed analysis for these problems is contained in the next two sections.

\section{Problem 1: Constant product demand}

Before finding solutions of the first problem, we make the following assumptions on the running cost function $h(x)$ and the random process $\alpha(t)$.

(A1) $h(x)$ is a convex function such that for positive constants $C_{h}$ and $k_{h}$,

$$
0 \leq h(x) \leq C_{h}\left(1+|x|^{k_{h}}\right) \text { and } h(x)>h(0)=0 \text { for all } x \neq 0 .
$$

Moreover, there exists a constant $c_{h}>0$ such that

$$
\frac{h_{x^{+}}\left(x_{2}\right)-h_{x^{+}}\left(x_{1}\right)}{x_{2}-x_{1}} \geq c_{h} \text { for all }-\left|\alpha_{2}-z\right| T \leq x_{1}<0 \leq x_{2} \leq\left|\alpha_{2}-z\right| T,
$$

where $h_{x^{+}}(x)$ denotes the right-hand derivative of $h(x)$.

(A2) The capacity process $\alpha(t) \in \mathcal{M}$ is a two state Markov chain governed by

$$
L_{\alpha} f(\cdot)(i)= \begin{cases}\lambda\left(f\left(\alpha_{2}\right)-f\left(\alpha_{1}\right)\right) & \text { if } i=\alpha_{1} \\ 0 & \text { if } i=\alpha_{2}\end{cases}
$$

for any function $f$ on $\mathcal{M}$. Here $\lambda \geq 0$ is the machine breakdown rate.

Examples of $h(x)$. Let us give a few examples of $h(x)$ that satisfy Assumption (A1).

(1) $h(x)=x^{2}$;

(2) $h(x)=h^{+} \max \{0, x\}+h^{-} \max \{0,-x\}$ where $h^{+}>0$ and $h^{-}>0$ are constants. This cost function was employed in [1].

(3) $h(x)$ is convex and piecewise linear with $h(0)=0, h_{x^{-}}(0)<0$, and $h_{x^{+}}(0)>0$.

Remark. Assumption (A2) is a condition on the machine capacity process $\alpha(t)$. It indicates that once the machine goes down it will never go up again. Such a situation occurs when the repairing is very expensive, or no repair facilities are available. As a result, replacement is a better alternative than repair. 
We first present a technical lemma that concerns with the running cost function $h(x)$. Since the lemma can be obtained easily from elementary properties of convex functions, the proof is omitted. Lemma 3.0. Under Assumption (A1), $h(x)$ is strictly increasing on $[0, \infty)$ and strictly decreasing on $(-\infty, 0]$. Moreover, for all $x>0, h_{x^{+}}(x) \geq h_{x^{-}}(x)>0$ and for all $x<0, h_{x^{-}}(x) \leq h_{x^{+}}(x)<0$, where $h_{x^{-}}(x)$ denotes the left-hand derivative of $h(x)$.

Next, we make precise definition on a class of admissible controls.

Definition. A control $u .=\left\{u_{t}: t \geq 0\right\}$ is admissible if $u_{t}$ is an $\mathcal{F}_{t}=\sigma\{\alpha(s), s \leq t\}$ adapted measurable process and $0 \leq u_{t} \leq \alpha(t)$ for all $0 \leq t \leq T$. $\mathcal{A}$ will denote the set of all admissible controls in the sequel.

Let $v(s, x, \alpha)$ denote the value function of the problem, i.e.,

$$
v(s, x, \alpha)=\inf _{u \in \mathcal{A}} J(s, x, \alpha, u .), \text { for } \alpha \in \mathcal{M}
$$

In what follows, some preliminary results on the value functions $v(s, x, \alpha)$ are given first. These results are summarized in the next lemma.

\section{Lemma 3.1.}

1) The value functions $v(s, x, \alpha)$ are convex functions in $x$ for each $s \in[0, T]$ and $\alpha \in \mathcal{M}$.

2) $v(s, x, \alpha) \in C\left([0, T], R^{1}\right)$ are the only viscosity solutions (for definition, see e.g., [9]) to the following dynamic programming equations.

$$
\left\{\begin{array}{l}
0=v_{s}\left(s, x, \alpha_{1}\right)+\min _{0 \leq u \leq \alpha_{1}}\left[(u-z) v_{x}\left(s, x, \alpha_{1}\right)\right]+e^{-\rho s} h(x)+\lambda\left(v\left(s, x, \alpha_{2}\right)-v\left(s, x, \alpha_{1}\right)\right), \\
0=v\left(T, x, \alpha_{1}\right)
\end{array}\right.
$$

and

$$
\left\{\begin{array}{l}
0=v_{s}\left(s, x, \alpha_{2}\right)+\min _{0 \leq u \leq \alpha_{2}}\left[(u-z) v_{x}\left(s, x, \alpha_{2}\right)\right]+e^{-\rho s} h(x) \\
0=v\left(T, x, \alpha_{2}\right) .
\end{array}\right.
$$

Proof. Under Assumption (A1), it is clear that $J(s, \cdot, \alpha, \cdot)$ is jointly convex in $(x, u$. ) for each $(s, \alpha)$. As a consequence, the value function $v(s, \cdot, \alpha)$ is convex for each $(s, \alpha)$. It can be shown, via a straightforward modification of the proof of [10, Theorem 1.1], that the value function is a viscosity solution to (3.3) and (3.4). Then by [7, Theorem 2.5], they are the unique viscosity solutions.

In [9], turnpike sets were introduced and used to characterize the optimal inventory levels. Since the problem considered in [9] is associated with a discounted cost over an infinity horizon, the dynamic of the problem is time homogeneous. Therefore, the turnpike sets are constants in 
time. However, if the problem is evaluated in finite horizon, the dynamic will not be homogeneous anymore. In the following, we modify the turnpike definition given in [9] to incorporate the variation of the turnpike sets with the changes of time.

Definition. $\phi(s)$ and $\psi(s)$ are said to be the turnpike sets for $\alpha=\alpha_{1}$ and $\alpha=\alpha_{2}$, respectively, if for all $s \in[0, T]$,

$$
\begin{aligned}
v\left(s, \phi(s), \alpha_{1}\right) & =\min _{x} v\left(s, x, \alpha_{1}\right) \\
\text { and } \quad v\left(s, \psi(s), \alpha_{2}\right) & =\min _{x} v\left(s, x, \alpha_{2}\right), \text { respectively. }
\end{aligned}
$$

Remark. Under infinite horizon formulation, normally, turnpike sets are attractors for optimal trajectories, whereas in the current setting, the turnpike sets are switching curves. To be consistent with the usual notation, we still call these switching curves turnpike sets.

Let $u^{*}(t, x, \alpha)$ denote the feedback control defined as follows.

$$
\begin{aligned}
& \text { If } \alpha(t)=\alpha_{1} \text {, then } u^{*}\left(t, x, \alpha_{1}\right)= \begin{cases}0 & \text { if } x>\phi(t) \\
\dot{\phi}(t)+z & \text { if } x=\phi(t) \\
\alpha_{1} & \text { if } x<\phi(t) .\end{cases} \\
& \text { If } \alpha(t)=\alpha_{2} \text {, then } u^{*}\left(t, x, \alpha_{2}\right)= \begin{cases}0 & \text { if } x>\psi(t) \\
\alpha_{2} & \text { if } x \leq \psi(t) .\end{cases}
\end{aligned}
$$

We shall show in the following that $\phi(s)$ and $\psi(s)$ are absolute continuous and satisfy $0 \leq \dot{\phi}(s)+z \leq$ $\alpha_{1}$ and $\dot{\psi}(s)+z>\alpha_{2}$. Using these properties, it is easy to see that under the control policy $u_{t}^{*}=u^{*}\left(t, x_{t}, \alpha(t)\right)$, the ordinary differential equation

$$
\dot{x}_{t}^{*}=u^{*}\left(t, x_{t}^{*}, \alpha(t)\right)-z, x_{s}^{*}=x
$$

has a unique solution.

In the rest of this section, we devote our attention to showing that the control $u_{t}^{*}=u^{*}\left(t, x_{t}, \alpha(t)\right)$ given by (3.5) and (3.6) is optimal.

By virtue of the transformation $\tilde{v}\left(s, x, \alpha_{1}\right)=e^{-\lambda s} v\left(s, x, \alpha_{1}\right)$, together (3.3) and (3.4), the problem defined by (2.1) and (2.2) is equivalent to the problems below.

$$
\begin{array}{ll}
\min . & \int_{s}^{T} e^{-\lambda(t-s)}\left[e^{-\rho t} h\left(x_{t}\right)+\lambda v\left(t, x_{t}, \alpha_{2}\right)\right] d t \\
\text { s.t. } & \dot{x}_{t}=u_{t}-z, x_{s}=x, 0 \leq u_{t} \leq \alpha_{1}
\end{array}
$$


where $v\left(t, x_{t}, \alpha_{2}\right)$ can be obtained from the following problem:

$$
\begin{array}{ll}
\min . & \int_{s}^{T} e^{-\rho t} h\left(x_{t}\right) d t \\
\text { s.t. } & \dot{x}_{t}=u_{t}-z, x_{s}=x, 0 \leq u_{t} \leq \alpha_{2} .
\end{array}
$$

In order to carry out the analysis, we need to evaluate $v\left(s, x, \alpha_{2}\right)$ in the first place. If $\alpha(s)=\alpha_{2}$, by virtue of Assumption (A2) on $\alpha(t), \alpha(t) \equiv \alpha_{2}$ for $t \in[s, T]$. Therefore, the problem becomes a deterministic problem. If $\alpha_{2}$ were larger than $z$, the problem would become much simpler: one would only need to produce at the maximum speed if $x_{t}<0$ and produce nothing if $x_{t}>0$. However, in our case, such production policy is no longer optimal since the state $x_{t}$ cannot stay at $x=0$. Intuitively, if the initial value $x_{s} \leq 0$, then it is better to take $u_{t}=\alpha_{2}$ in order to keep $x_{t}(\leq 0)$ as close as possible to $x=0$ from below. If $x_{s} \geq z(T-s)$, then it is better to take $u_{t}=0$ so as to keep $x_{t}(\geq 0)$ as close as possible to $x=0$ from above. Let us now consider the case when $x_{s}$ is less than $z(T-s)$ but is close to $z(T-s)$. In this case, if we take $u_{t}=0$ at the beginning for a while, then switch the control to $u_{t}=\alpha_{2}$ before $x_{t}$ actually reaches 0 . It can be seen that in this way the cost will be smaller than the cost incurred if we use $u_{t}=0$ until $x_{t}=0$, then switch to $u_{t}=\alpha_{2}$. Therefore, there must be a curve, say $\psi(s) \geq 0$ (which will be seen to be a turnpike set), such that $u_{t}=0$ when $x_{t} \geq \psi(t)$ and $u_{t}=\alpha_{2}$ when $x_{t}<0$. Based on these observations, a crucial task is to identify this curve $\psi(s)$.

Let $s=0$ and $x_{0}=x$ which is smaller than $z T$ but close to $z T$. Then $u_{t}=0$ and $x_{t}=x-z t$ before it reaches $\psi(t)$. Let $0 \leq \eta \leq T$ be such that $x_{\eta}=x-z \eta=\psi(\eta)$. Then the incurred cost is given by

$$
J=\int_{0}^{\eta} e^{-\rho t} h(x-z t) d t+\int_{\eta}^{T} e^{-\rho t} h\left(x-z \eta+\left(\alpha_{2}-z\right)(t-\eta)\right) d t .
$$

Since $\psi(s)$ is so given that the cost needs to be minimized,

$$
0=\frac{\partial}{\partial \eta} J=\alpha_{2} \int_{\eta}^{T} e^{-\rho t} h_{x^{+}}\left(x-z \eta+\left(\alpha_{2}-z\right)(t-\eta)\right) d t .
$$

Note that $\psi(\eta)=x-z \eta$. The curve $\psi(s)$ has to satisfy

$$
\int_{\eta}^{T} e^{-\rho t} h_{x^{+}}\left(\psi(\eta)+\left(\alpha_{2}-z\right)(t-\eta)\right) d t=0
$$

Setting $\eta=s$, the curve $x=\psi(s)$ is given by

$$
\int_{s}^{T} e^{-\rho t} h_{x^{+}}\left(\psi(s)+\left(\alpha_{2}-z\right)(t-s)\right) d t=0 .
$$

The next two lemmas are concerned with properties of $\psi(s)$. 
Lemma 3.2. Let $\psi(s)$ be defined as in (3.9). Then $\psi(s)$ is a continuous function uniquely determined by (3.9) and satisfies

1) $0<\psi(s)<\left|\alpha_{2}-z\right|(T-s)$ for $s \in[0, T)$ and $\psi(T)=0$;

2) $\psi(s)$ is monotone decreasing and absolute continuous. Moreover, $\dot{\psi}(s)+z>\alpha_{2}$.

Lemma 3.3. Let $J(s, x)$ denote the cost function under the control defined by (3.6), i.e., $u_{t}^{*}=$ $u^{*}\left(t, x_{t}, \alpha_{2}\right)$, and $J(s, x)=J\left(s, x, \alpha_{2}, u^{*}.\right)$. Then $J(s, x)$ is continuously differentiable on $[0, T] \times R^{1}$ and satisfies the dynamic programming equation (3.4). Thus, $J(s, x)=v\left(s, x, \alpha_{2}\right)$. Moreover,

$$
v\left(s, \psi(s), \alpha_{2}\right)=J(s, \psi(s))=\min _{x} J(s, x)=\min _{x} v\left(s, x, \alpha_{2}\right) .
$$

Since the proofs of these lemmas are rather technical, we postpone them until $\S 5$.

Remark. The absolute continuity of $\psi(s)$ implies that it is differentiable almost everywhere in $s$. $\dot{\psi}(s)+z>\alpha_{2}$ says that if $x_{t} \leq \psi(t)$ for some $t_{1}$, then $x_{t}$ will stay below $\psi(t)$ for all $t_{1} \leq t \leq T$.

We now turn to the problem in (3.7). Let

$$
H(s, x)=h(x)+\lambda e^{\rho s} v\left(s, x, \alpha_{2}\right) .
$$

Then,

$$
J\left(s, x, \alpha_{1}, u \cdot\right)=\int_{s}^{T} e^{-\lambda(t-s)} e^{-\rho t} H\left(t, x_{t}\right) d t .
$$

Note that $H(s, x)$ is convex in $x$ for each $s$. We are to show that the turnpike set for $\alpha=\alpha_{1}$ is given by the minimizer of $H(s, x)$, i.e.,

$$
H(s, \phi(s))=\min _{x} H(s, x)
$$

Moreover, the control given in (3.5) is optimal for $\alpha=\alpha_{1}$. To proceed, we need to consider an important property possessed by $\phi(s)$, which is described in the following definition.

Definition. A function given by $x=\gamma(s)$ is said to be traceable on $[0, T]$ if $\gamma(s)$ is absolutely continuous on $[0, T]$ and $0 \leq \dot{\gamma}(s)+z \leq z$, a.e. in $s \in[0, T]$.

It is easy to see that a traceable curve is always decreasing.

By standard real analysis theory, any absolutely continuous function $\gamma(s)$ is differentiable a.e. and $\int_{a}^{b} \dot{\gamma}(s) d s=\gamma(b)-\gamma(a)$ for any $a$ and $b$. If a function $\gamma(s)$ is traceable, then there exists a control $0 \leq u_{s}=\dot{\gamma}(s)+z \leq z<\alpha_{1}$ such that the corresponding system trajectory $x_{t}$ may stay on the curve $\gamma(s)$ after it reaches $\gamma(s)$.

The next lemma is concerned with the traceability of $\phi(s)$. Its proof will be provided in $\S 5$. 
Lemma 3.4. Let $\phi(s)$ be the minimizer of $H(s, x)$. Then $\phi(s)$ is a single-valued function and satisfies:

1) $0 \leq \phi(s)<\psi(s)$ for $s \in[0, T)$ and $\phi(T)=0$;

2) $\phi(s)$ is traceable.

Let

$$
\tilde{H}(s, x)=h(x)+\lambda e^{\rho s} \int_{s}^{T} e^{-\rho t} h\left(x+\left(\alpha_{2}-z\right)(t-s)\right) d t .
$$

Note that, by Lemma $3.4, \phi(s)<\psi(s)$ and

$$
v\left(s, x, \alpha_{2}\right)=\int_{s}^{T} e^{-\rho t} h\left(x+\left(\alpha_{2}-z\right)(t-s)\right) d t \text { if } x \leq \psi(s) .
$$

It follows that

$$
\min _{x} H(s, x)=\min _{x} \tilde{H}(s, x)=h(\phi(s))+\lambda e^{\rho s} \int_{s}^{T} e^{-\rho t} h\left(\phi(s)+\left(\alpha_{2}-z\right)(t-s)\right) d t .
$$

It can also be seen that $\phi(s)$ is the only solution to (3.12). In the rest of the paper, we shall use (3.12) as the definition of $\phi(s)$.

Theorem 3.1. Let $\phi(s)$ and $\psi(s)$ be given as in (3.1.) and (3.9), respectively. Then $\phi(s)$ and $\psi(s)$ are the turnpike sets for $\alpha=\alpha_{1}$ and $\alpha=\alpha_{2}$, respectively. Moreover, the feedback control $u_{t}^{*}=u^{*}\left(t, x_{t}^{*}, \alpha(t)\right)$ given in (3.5) and (3.6) is optimal.

Proof. Lemma 3.3 indicates that $\psi(s)$ is the turnpike set for $\alpha=\alpha_{2}$, and $u_{t}^{*}=u^{*}\left(t, x_{t}, \alpha_{2}\right)$ is optimal for $\alpha=\alpha_{2}$.

Recall that

$$
v\left(s, x, \alpha_{1}\right)=\inf _{0 \leq u . \leq \alpha_{1}} \int_{s}^{T} e^{-\lambda(t-s)} e^{-\rho t} H\left(t, x_{t}\right) d t .
$$

Since $\phi(s)$ is traceable and it is the minimizer of $H(s, x)$,

$$
v\left(s, \phi(s), \alpha_{1}\right)=\int_{s}^{T} e^{-\lambda(t-s)} e^{-\rho t} H(t, \phi(t)) d t .
$$

Moreover,

$$
v\left(s, x, \alpha_{1}\right) \geq v\left(s, \phi(s), \alpha_{1}\right) \text { for all } x .
$$

Thus,

$$
v\left(s, \phi(s), \alpha_{1}\right)=\min _{x} v\left(s, x, \alpha_{1}\right) .
$$

This indicates that $\phi(s)$ is the turnpike set for $\alpha=\alpha_{1}$. 
Let $\tau$ denote the machine breakdown time and let $u_{t}=\left\{\begin{array}{ll}u_{t}^{1} & \text { if } t<\tau \\ u_{t}^{2} & \text { if } t \geq \tau\end{array}\right.$ denote an arbitrary control with $0 \leq u_{t}^{1} \leq \alpha_{1}$ and $0 \leq u_{t}^{2} \leq \alpha_{2}$, and $x_{t}^{*}$ is the trajectory under $u_{t}^{*}$. Then

$$
\begin{aligned}
J\left(s, x, \alpha_{1}, u .\right) & =\int_{s}^{T} e^{-\lambda(t-s)}\left[e^{-\rho t} h\left(x_{t}\right)+\lambda v\left(t, x_{t}, \alpha_{2}\right)\right] d t \\
& \geq \int_{s}^{T} e^{-\lambda(t-s)}\left[H\left(t, x_{t}^{*}\right)\right] d t \\
& =v\left(s, x, \alpha_{1}\right),
\end{aligned}
$$

where $u_{t}^{*}=u^{*}\left(t, x_{t}^{*}, \alpha(t)\right)$. The last inequality follows from the fact that the control $u_{t}^{*}$ makes the corresponding state trajectory $x_{t}^{*}$ go to $\phi(s)$ in the fastest way, i.e., $H\left(t, x_{t}^{*}\right) \leq H\left(t, x_{t}\right)$. This completes the proof.

Using the control given in (3.5), the value function $v\left(s, x, \alpha_{1}\right)$ can be written as

$$
v\left(s, x, \alpha_{1}\right)=\left\{\begin{array}{c}
\int_{s}^{T} e^{-\lambda(t-s)}\left[e^{-\rho t} h(x-z(t-s))+\lambda v\left(t, x-z(t-s), \alpha_{2}\right)\right] d t \\
\text { if } x \geq z(T-s) \\
\int_{s}^{s_{1}} e^{-\lambda(t-s)}\left[e^{-\rho t} h(x-z(t-s))+\lambda v\left(t, x-z(t-s), \alpha_{2}\right)\right] d t \\
+\int_{s_{1}}^{T} e^{-\lambda(t-s)}\left[e^{-\rho t} h(\phi(t))+\lambda v\left(t, \phi(t), \alpha_{2}\right)\right] d t \\
\text { if } \phi(s)<x<z(T-s) \\
\int_{s}^{T} e^{-\lambda(t-s)}\left[e^{-\rho t} h(\phi(t))+\lambda v\left(t, \phi(t), \alpha_{2}\right)\right] d t \\
\text { if } x=\phi(s) \\
\int_{s}^{s_{2}} e^{-\lambda(t-s)}\left[e^{-\rho t} h\left(x+\left(\alpha_{1}-z\right)(t-s)\right)+\lambda v\left(t, x+\left(\alpha_{1}-z\right)(t-s), \alpha_{2}\right)\right] d t \\
+\int_{s_{2}}^{T} e^{-\lambda(t-s)}\left[e^{-\rho t} h(\phi(t))+\lambda v\left(t, \phi(t), \alpha_{2}\right)\right] d t \\
\text { if }-\left(\alpha_{1}-z\right)(T-s)<x<\phi(s) \\
\int_{s}^{T} e^{-\lambda(t-s)}\left[e^{-\rho t} h\left(x+\left(\alpha_{1}-z\right)(t-s)\right)+\lambda v\left(t, x+\left(\alpha_{1}-z\right)(t-s), \alpha_{2}\right)\right] d t \\
\text { if } x \leq-\left(\alpha_{1}-z\right)(T-s),
\end{array}\right.
$$

where $s_{1}$ is the first time that $x-z(t-s)$ hits $\phi(t)$ and $s_{2}$ is the first time that $x+\left(\alpha_{1}-z\right)(t-s)$ hits $\phi(t)$, respectively. Thus,

$$
x-z\left(s_{1}-s\right)=\phi\left(s_{1}\right) \text { and } x+\left(\alpha_{1}-z\right)\left(s_{2}-s\right)=\phi\left(s_{2}\right), \text { respectively. }
$$


Using the control (3.6), we can write the value function $v\left(s, x, \alpha_{2}\right)$ as follows:

$$
v\left(s, x, \alpha_{2}\right)=\left\{\begin{array}{lc}
\int_{s}^{T} e^{-\rho t} h(x-z(t-s)) d t & \text { if } x \geq z(T-s) \\
\int_{s}^{s_{0}} e^{-\rho t} h(x-z(t-s)) d t+\int_{s_{0}}^{T} e^{-\rho t} h\left(x-z\left(s_{0}-s\right)+\left(\alpha_{2}-z\right)\left(t-s_{0}\right)\right) d t \\
\int_{s}^{T} e^{-\rho t} h\left(x+\left(\alpha_{2}-z\right)(t-s)\right) d t & \text { if } \psi(s)<x<z(T-s) \\
& \text { if } x \leq \psi(s)
\end{array}\right.
$$

where $s_{0}$ is the first time that $x-z(t-s)$ hits $\psi(t)$. Thus, $x-z\left(s_{0}-s\right)=\psi\left(s_{0}\right)$.

Example 3.1. In this example, the cost function is given by $h(x)=h^{+} \max \{0, x\}+h^{-} \max \{0,-x\}$.

Then, (3.9) becomes

$$
\int_{s}^{t_{1}} e^{-\rho t} h^{+} d t-\int_{t_{1}}^{T} e^{-\rho t} h^{-} d t=0
$$

where $t_{1}$ is given by $\psi(s)+\left(\alpha_{2}-z\right)\left(t_{1}-s\right)=0$. This yields

$$
\psi(s)=\frac{\alpha_{2}-z}{\rho} \log \frac{h^{+}+h^{-} e^{-\rho(T-s)}}{h^{+}+h^{-}} .
$$

We now identify $\phi(s)$. Recall that $0 \leq \phi(s)<\psi(s) \leq\left|\alpha_{2}-z\right|(T-s)$ and for $x<\psi(s)$,

$$
v\left(s, x, \alpha_{2}\right)=\int_{s}^{T} e^{-\rho t} h\left(x+\left(\alpha_{2}-z\right)(t-s)\right) d t .
$$

Moreover, for $0 \leq x \leq\left|\alpha_{2}-z\right|(T-s)$,

$$
\begin{aligned}
& v\left(s, x, \alpha_{2}\right) \\
= & e^{-\rho s} \int_{0}^{\frac{x}{\alpha_{2}-z}} e^{-\rho t} h^{+}\left(x+\left(\alpha_{2}-z\right) t\right) d t-\int_{\frac{x}{\left|\alpha_{2}-z\right|}}^{T-s} e^{-\rho t} h^{-}\left(x+\left(\alpha_{2}-z\right) t\right) d t \\
= & e^{-\rho s} h^{+} \rho^{-1}\left[x+\left(\alpha_{2}-z\right) \rho^{-1}\left(1-e^{-\rho x /\left|\alpha_{2}-z\right|}\right)\right] \\
& +h^{-} \rho^{-1}\left[\left(x+\left(\alpha_{2}-z\right)(T-s) e^{-\rho(T-s)}+\left|\alpha_{2}-z\right| \rho^{-1}\left(e^{-\rho x /\left|\alpha_{2}-z\right|}-e^{-\rho(T-s)}\right)\right]\right.
\end{aligned}
$$

This together with (3.12) yields

$$
\phi(s)=\max \left\{0,-\frac{\left|\alpha_{2}-z\right|}{\rho} \log \frac{\rho h^{+}+\lambda\left(h^{+}+h^{-} e^{-\rho(T-s)}\right)}{\lambda\left(h^{+}+h^{-}\right)}\right\} .
$$

Equivalently, $\phi(s)$ can also be written as:

$$
\phi(s)=0 \text { for all } s \in[0, T] \text { if } \lambda h^{-}-\rho h^{+} \leq 0
$$


otherwise,

$$
\phi(s)=\left\{\begin{array}{ll}
-\frac{\left|\alpha_{2}-z\right|}{\rho} \log \frac{\rho h^{+}+\lambda\left(h^{+}+h^{-} e^{-\rho(T-s)}\right)}{\lambda\left(h^{+}+h^{-}\right)} & \text {if } s \leq T+\rho^{-1} \log \frac{\lambda h^{-}-\rho h^{+}}{\lambda h^{-}} \\
0 & \text { if } s>T+\rho^{-1} \log \frac{\lambda h^{-}-\rho h^{+}}{\lambda h^{-}} .
\end{array} .\right.
$$

As $T \rightarrow \infty$, it is easily seen that

$$
\phi(s) \rightarrow \max \left\{0,-\frac{\left|\alpha_{2}-z\right|}{\rho} \log \frac{\left(\rho h^{+}+\lambda h^{+}\right)}{\lambda\left(h^{+}+h^{-}\right)}\right\},
$$

which provides the same turnpike as in [1].

\section{Problem 2: Constant machine capacity}

(A1') Let Assumption (A1) be satisfied with (3.1) replaced by

$$
\frac{h_{x^{+}}\left(x_{2}\right)-h_{x^{+}}\left(x_{1}\right)}{x_{2}-x_{1}} \geq c_{h} \text { for all }-\left|\alpha_{0}-z_{2}\right| T \leq x_{1}<0 \leq x_{2} \leq\left|\alpha_{0}-z_{2}\right| T,
$$

with $z_{2}$ given below.

(A2') The demand process $z_{t} \in \mathcal{Z}$ is also a two state Markov chain governed by

$$
L_{z} f(\cdot)(i)= \begin{cases}\lambda^{\prime}\left(f\left(z_{2}\right)-f\left(z_{1}\right)\right) & \text { if } i=z_{1} \\ 0 & \text { if } i=z_{2}\end{cases}
$$

for any function $f$ on $\mathcal{Z}$.

Let $v(s, x, z)$ denote the value function of the problem, i.e.,

$$
v(s, x, z)=\inf _{u \in \mathcal{A}} J(s, x, z, u .), \text { for } z \in \mathcal{Z} \text {. }
$$

The next lemma is an analog of Lemma 3.1. Since all the results in this section can be proved similarly as in the last section, the proofs will be omitted.

\section{Lemma 4.1.}

1) The value functions $v(s, x, z)$ are convex functions in $x$ for each $s \in[0, T]$ and $z \in \mathcal{Z}$.

2) $v(s, x, z) \in C\left([0, T], R^{1}\right)$ are the only viscosity solutions the following dynamic programming equations.

$$
\left\{\begin{array}{l}
0=v_{s}\left(s, x, z_{1}\right)+\min _{0 \leq u \leq \alpha_{0}}\left[\left(u-z_{1}\right) v_{x}\left(s, x, z_{1}\right)\right]+e^{-\rho s} h(x)+\lambda^{\prime}\left(v\left(s, x, z_{2}\right)-v\left(s, x, z_{1}\right)\right), \\
0=v\left(T, x, z_{1}\right)
\end{array}\right.
$$


and

$$
\left\{\begin{array}{l}
0=v_{s}\left(s, x, z_{2}\right)+\min _{0 \leq u \leq \alpha_{0}}\left[\left(u-z_{2}\right) v_{x}\left(s, x, z_{2}\right)\right]+e^{-\rho s} h(x) \\
0=v\left(T, x, z_{2}\right)
\end{array}\right.
$$

Definition. $\phi(s)$ and $\psi(s)$ are said to be turnpike sets if

$$
\begin{aligned}
v\left(s, \phi(s), z_{1}\right) & =\min _{x} v\left(s, x, z_{1}\right) \\
\text { and } \quad v\left(s, \psi(s), z_{2}\right) & =\min _{x} v\left(s, x, z_{2}\right) .
\end{aligned}
$$

Let $\hat{H}(s, x)=h(x)+\lambda \int_{s}^{T} e^{-\rho t} h\left(x+\left(\alpha_{0}-z_{2}\right)(t-s)\right) d t$. Then $\psi(s)$ and $\phi(s)$ are determined by:

$$
\int_{s}^{T} e^{-\rho t} h_{x^{+}}\left(\psi(s)+\left(\alpha_{0}-z_{2}\right)(t-s)\right) d t=0
$$

and

$$
\hat{H}(s, \phi(s))=\min _{x} \hat{H}(s, x)
$$

Lemma 4.2. $\phi(s)$ and $\psi(s)$ are single-valued absolute continuous functions. They satisfy the following properties:

(1) $0<\psi(s)<\left|\alpha_{0}-z_{2}\right|(T-s)$ for $s \in[0, T)$ and $\psi(T)=0$;

(D) $\psi(s)$ is monotone decreasing and $\dot{\psi}(s)+z_{2}>\alpha_{0}$;

(3) $0 \leq \phi(s)<\psi(s)$ for $s \in[0, T)$ and $\phi(T)=0$;

(4) $\phi(s)$ is monotone decreasing and $\dot{\phi}(s)+z_{1} \geq \alpha_{0}-z_{2}+z_{1}$.

Note that by (4) of the above lemma, a sufficient condition for $\phi(s)$ to be traceable (i.e., $0 \leq$ $\left.\dot{\phi}(s)+z_{1} \leq \alpha_{0}\right)$ is $z_{1} \geq z_{2}-\alpha_{0}$. Consider related problems in tracking parameter variations of a time varying system (cf. [2, pp. 123]). In such problems, the system parameter processes cannot be changing too fast and the signal to noise ratio has to be kept in a reasonable range, i.e., an identifiability or tracking capability condition needs to be satisfied. It seems that the problem discussed in this section is similar to the tracking parameter variation problems since the total demand process can be regarded as a parameter process and the main task is to choose control variables to make the total finished goods as close to the total demand as possible. In our problem, the condition $z_{1} \geq z_{2}+\alpha_{0}$ guarantees the volatility of the total demand to be confined in a certain domain so as to assure the traceability.

Theorem 4.1. Suppose that (A1'), (A') are satisfied, and $z_{1} \geq z_{2}-\alpha_{0}$. Let $u^{*}\left(t, x_{t}, z\right)$ be defined 
as follows:

$$
u^{*}(t, x, z)= \begin{cases}u^{*}\left(t, x, z_{1}\right)= \begin{cases}0 & \text { if } x>\phi(t) \\ \dot{\phi}(t)+z_{1} & \text { if } x=\phi(t) \\ \alpha_{0} & \text { if } x<\phi(t)\end{cases} \\ u^{*}\left(t, x, z_{2}\right)= \begin{cases}0 & \text { if } x>\psi(t) \\ \alpha_{0} & \text { if } x \leq \psi(t) .\end{cases} \end{cases}
$$

Then under the control $u_{t}^{*}=u^{*}\left(t, x_{t}, z_{t}\right)$, the equation

$$
\dot{x}_{t}^{*}=u^{*}\left(t, x_{t}^{*}, z_{t}\right)-z_{t}, x_{0}^{*}=x
$$

has a unique solution. Therefore, the control $u_{t}^{*}$ is optimal.

Example 4.1. We consider the cost $h(x)=h^{+} \max \{0, x\}+h^{-} \max \{0,-x\}$. Then,

$$
\begin{gathered}
\psi(s)=\frac{\alpha_{0}-z_{2}}{\rho} \log \frac{h^{+}+h^{-} e^{-\rho(T-s)}}{h^{+}+h^{-}} . \\
\phi(s)=0 \text { if } \lambda h^{-}-\rho h^{+} \leq 0 .
\end{gathered}
$$

If $\lambda h^{-}-\rho h^{+}>0$, then

$$
\phi(s)=\left\{\begin{array}{ll}
-\frac{\left|\alpha_{0}-z_{2}\right|}{\rho} \log \frac{\rho h^{+}+\lambda\left(h^{+}+h^{-} e^{-\rho(T-s)}\right)}{\lambda\left(h^{+}+h^{-}\right)} & \text {if } s \leq T+\rho^{-1} \log \frac{\lambda h^{-}-\rho h^{+}}{\lambda h^{-}} \\
0 & \text { if } s>T+\rho^{-1} \log \frac{\lambda h^{-}-\rho h^{+}}{\lambda h^{-}}
\end{array} .\right.
$$

Remark. Note that the assumption $z_{1} \geq z_{2}-\alpha_{0}$ in Theorem 4.1 is a relatively conservative one. In Example 4.1, this condition can be relaxed to

$$
z_{1} \geq\left|\alpha_{0}-z_{2}\right| \frac{\lambda h^{-}-\rho h^{+}}{\lambda\left(h^{+}+h^{-}\right)} .
$$

It can be seen that (4.7) is also necessary for the traceability of $\phi(s)$ in this example. If (4.7) fails. $\phi(s)$ will no longer be the turnpike of the problem since it is not traceable on $[0, T]$ anymore. Let $\tilde{\phi}(s)$ denote the turnpike set for $\alpha=\alpha_{1}$ defined as $v\left(s, \tilde{\phi}(s), z_{1}\right)=\min _{x} v\left(s, x, z_{1}\right)$. Then, similarly to Lemma $4.2,0 \leq \tilde{\phi}(s) \leq \psi(s)$. It can be shown that $h(x)+\lambda v\left(s, x, z_{2}\right)$ is strictly convex on $[0, \psi(s)]$, which implies that $v\left(s, x, z_{1}\right)$ is also strictly convex on $[0, \psi(s)]$ (see [9] for details). Therefore, $\tilde{\phi}(s)$ is a continuous function. Intuitively, the optimal control $u^{*}\left(t, x, \alpha_{1}\right)$ should be given as in (4.6) with $\tilde{\phi}(s)$ in place of $\phi(s)$ provided that $\tilde{\phi}(s)$ is traceable. Let $s_{0}=T+\rho^{-1} \log \frac{\lambda h^{-}-\rho h^{+}}{\lambda h^{-}}$. Then, $0 \leq \dot{\phi}(s)+z_{1} \leq \alpha_{0}$ for $s \geq s_{0}$. This implies $\tilde{\phi}(s)=\phi(s)$ for $s \geq s_{0}$. However, if $0>s_{0}>T$ and $z_{2}$ is large enough, the traceability property of $\tilde{\phi}(s)$ will not hold, which makes the problem very complicated. Explicit optimal solution is very difficult to obtain. 


\section{Proofs of results}

In this section, proofs of Lemmas 3.2, 3.3, and 3.4 will be presented.

Proof of Lemma 3.2. Let $\psi(s)$ be defined as in (3.9). The proof of Lemma 3.2 is divided into several steps.

(1) $0<\psi(s)<\left|\alpha_{2}-z\right|(T-s)$ for $s \in[0, T)$.

If $\psi(s) \leq 0$, for some $s \in[0, T)$, then

$$
\int_{s}^{T} e^{-\rho t} h_{x^{+}}\left(\psi(s)+\left(\alpha_{2}-z\right)(t-s)\right) d t \leq \int_{s}^{T} e^{-\rho t} h_{x^{+}}\left(\left(\alpha_{2}-z\right)(t-s)\right) d t<0,
$$

since $\left(\alpha_{2}-z\right)(t-s)<0$ for $t \in[s, T]$ and $h_{x^{+}}(x)<0$ for $x<0$, which contradicts (3.9). This implies that $\psi(s)>0$ for $s \in[0, T)$.

Similarly, it can be shown that $\psi(s)<\left|\alpha_{2}-z\right|(T-s)$ for $s \in[0, T)$.

(2) $\psi(s)$ is a continuous function.

If there exist $\psi_{1}(s)<\psi_{2}(s)$ for some $s \in[0, T]$ such that

$$
\int_{s}^{T} e^{-\rho t} h_{x^{+}}\left(\psi_{i}(s)+\left(\alpha_{2}-z\right)(t-s)\right) d t=0 \text { for } i=1,2 .
$$

Then, following from the monotonicity of $h_{x^{+}}$, we have

$$
h_{x^{+}}\left(\psi_{1}(s)+\left(\alpha_{2}-z\right)(t-s)\right)=h_{x^{+}}\left(\psi_{2}(s)+\left(\alpha_{2}-z\right)(t-s)\right) \text {, a.e. } t \in[s, T] \text {. }
$$

Let $t_{0}=s+\frac{\psi_{1}(s)+\psi_{2}(s)}{2\left|\alpha_{2}-z\right|}$. Then,

$$
t_{0} \in[s, T] \text { and } \frac{\psi_{1}(s)+\psi_{2}(s)}{2}+\left(\alpha_{2}-z\right)\left(t_{0}-s\right)=0 .
$$

There exists a $\delta_{0}>0$ such that

$$
\begin{aligned}
\psi_{1}(s)+\left(\alpha_{2}-z\right)(t-s) & <0 \text { on }\left[t_{0}-\delta_{0}, t_{0}+\delta_{0}\right] \cap[s, T] \\
\text { and } \quad \psi_{2}(s)+\left(\alpha_{2}-z\right)(t-s) & >0 \text { on }\left[t_{0}-\delta_{0}, t_{0}+\delta_{0}\right] \cap[s, T] .
\end{aligned}
$$

This leads to

$$
\begin{array}{ll} 
& h_{x^{+}}\left(\psi_{1}(s)+\left(\alpha_{2}-z\right)(t-s)\right)<0 \text { on }\left[t_{0}-\delta_{0}, t_{0}+\delta_{0}\right] \cap[s, T] \\
\text { and } \quad & h_{x^{+}}\left(\psi_{2}(s)+\left(\alpha_{2}-z\right)(t-s)\right)>0 \text { on }\left[t_{0}-\delta_{0}, t_{0}+\delta_{0}\right] \cap[s, T]
\end{array}
$$

But this contradicts (5.1). Therefore, $\psi(s)$ is a single-valued function. 
Let $F(s, x)=\int_{s}^{T} e^{-\rho t} h_{x^{+}}\left(x+\left(\alpha_{2}-z\right)(t-s)\right) d t$. Then $F(s, x)$ is continuous in $(s, x)$. This yields the continuity of $\psi(s)$.

(3) $\psi(s)$ is monotone decreasing and absolute continuous.

By means of changing variables, we rewrite (3.9) as follows:

$$
\int_{\psi(s)+\left(\alpha_{2}-z\right)(T-s)}^{\psi(s)} \exp \left(-\frac{\rho t}{\alpha_{2}-z}\right) h_{x^{+}}(t) d t=0 \text { for all } s \in[0, T]
$$

If there exist $0 \leq s_{1}<s_{2} \leq T$ such that $\psi\left(s_{1}\right)<\psi\left(s_{2}\right)$, then

$$
\psi\left(s_{1}\right)+\left(\alpha_{2}-z\right)\left(T-s_{1}\right)<\psi\left(s_{2}\right)+\left(\alpha_{2}-z\right)\left(T-s_{2}\right) \leq 0<\psi\left(s_{1}\right)<\psi\left(s_{2}\right) .
$$

This together with (5.2) implies that

$$
\int_{\psi\left(s_{1}\right)+\left(\alpha_{2}-z\right)\left(T-s_{1}\right)}^{\psi\left(s_{2}\right)+\left(\alpha_{2}-z\right)\left(T-s_{2}\right)} \exp \left(-\frac{\rho t}{\alpha_{2}-z}\right) h_{x^{+}}(t) d t=\int_{\psi\left(s_{1}\right)}^{\psi\left(s_{2}\right)} \exp \left(-\frac{\rho t}{\alpha_{2}-z}\right) h_{x^{+}}(t) d t .
$$

Since $h_{x^{+}}(t)<0$ for $t \in\left[\psi\left(s_{1}\right)+\left(\alpha_{2}-z\right)\left(T-s_{1}\right), \psi\left(s_{2}\right)+\left(\alpha_{2}-z\right)\left(T-s_{2}\right)\right]$ and $h_{x^{+}}(t)>0$ for $t \in\left[\psi\left(s_{1}\right), \psi\left(s_{2}\right)\right],(5.3)$ cannot hold. The contradiction infers that $\psi(s)$ is a decreasing function.

(4) $\dot{\psi}(s)>\alpha_{2}-z$.

Differentiating $(5.2)$ with respect to $s$, we have

$$
\begin{aligned}
& \dot{\psi}(s) \exp \left(-\frac{\rho \psi(s)}{\alpha_{2}-z}\right) h_{x^{+}}(\psi(s)) \\
& -\left(\dot{\psi}(s)-\left(\alpha_{2}-z\right)\right) \exp \left(-\frac{\rho\left(\psi(s)+\left(\alpha_{2}-z\right)(T-s)\right)}{\alpha_{2}-z}\right) h_{x}\left(\psi(s)+\left(\alpha_{2}-z\right)(T-s)\right)=0 .
\end{aligned}
$$

This gives

$$
\dot{\psi}(s)-\left(\alpha_{2}-z\right)=\frac{\alpha_{2}-z \mid h_{x^{+}}(\psi(s))}{h_{x^{+}}(\psi(s))-\exp (-\rho(T-s)) h_{x^{+}}\left(\psi(s)+\left(\alpha_{2}-z\right)(T-s)\right)}>0 \text { a.e. in }[0, T] .
$$

This equality also implies that $\psi(s)$ is absolute continuous.

Proof of Lemma 3.3. It suffices to show that the cost function $J(s, x)$ under the control given in (3.6) is continuously differentiable and satisfies Equation (3.4).

(1) If $x \leq \psi(s)$, then $u_{t}^{*}=u^{*}\left(t, x_{t}, \alpha_{2}\right)=\alpha_{2}$. Thus,

$$
J(s, x)=\int_{s}^{T} e^{-\rho t} h\left(x+\left(\alpha_{2}-z\right)(t-s)\right) d t .
$$

The partial derivatives of $J(s, x)$ with respect to $s$ and $x$ are given by

$$
\begin{aligned}
J_{s}(s, x) & =-e^{-\rho s} h(x)-\left(\alpha_{2}-z\right) \int_{s}^{T} e^{-\rho t} h_{x^{+}}\left(x+\left(\alpha_{2}-z\right)(t-s)\right) d t \\
J_{x}(s, x) & =\int_{s}^{T} e^{-\rho t} h_{x^{+}}\left(x+\left(\alpha_{2}-z\right)(t-s)\right) d t \\
& \leq \int_{s}^{T} e^{-\rho t} h_{x^{+}}\left(\psi(s)+\left(\alpha_{2}-z\right)(t-s)\right) d t \\
& =0
\end{aligned}
$$


Therefore,

$$
\begin{aligned}
& J_{s}(s, x)+\min _{0 \leq u \leq \alpha_{2}}(u-z) J_{x}(s, x)+e^{-\rho s} h(x) \\
= & J_{s}(s, x)+\left(\alpha_{2}-z\right) J_{x}(s, x)+e^{-\rho s} h(x) \\
= & 0 .
\end{aligned}
$$

(2) If $x \geq z(T-s)$, then $u_{t}^{*}=u^{*}\left(t, x_{t}, \alpha_{2}\right)=0$ since $x_{t}$ will stay above $z(T-t)$ for all $t \in[s, T]$. Thus,

$$
J(s, x)=\int_{s}^{T} e^{-\rho t} h(x-z(t-s)) d t .
$$

As a result,

$$
\begin{aligned}
J_{s}(s, x) & =-e^{-\rho s} h(x)+z \int_{s}^{T} e^{-\rho t} h_{x^{+}}(x-z(t-s)) d t \\
J_{x}(s, x) & =\int_{s}^{T} e^{-\rho t} h_{x^{+}}(x-z(t-s)) d t \\
& \geq \int_{s}^{T} e^{-\rho t} h_{x^{+}}(z(T-s)-z(t-s)) d t \\
& \geq 0 .
\end{aligned}
$$

Therefore,

$$
\begin{aligned}
& J_{s}(s, x)+\min _{0 \leq u \leq \alpha_{2}}(u-z) J_{x}(s, x)+e^{-\rho s} h(x) \\
= & J_{s}(s, x)-z J_{x}(s, x)+e^{-\rho s} h(x) \\
= & 0 .
\end{aligned}
$$

(3) If $\psi(s)<x<z(T-s)$, then there exists $s<\eta<T$ such that $x-z(\eta-s)=\psi(\eta)$. The optimal control is $u_{t}^{*}=0$ for $t \leq \eta$ and $u_{t}^{*}=\alpha_{2}$ for $t>\eta$. Thus,

$$
J(s, x)=\int_{s}^{\eta} e^{-\rho t} h(x-z(t-s)) d t+\int_{\eta}^{T} e^{-\rho t} h\left(x-z(\eta-s)+\left(\alpha_{2}-z\right)(t-\eta)\right) d t .
$$

Note that $\eta=\eta(s, x)$. For each fixed $s, \eta$ is increasing in $x$. Moreover, for each fixed $x, \eta$ is increasing in $s$. Hence,

$$
\begin{aligned}
J_{s}(s, x)= & -e^{-\rho s} h(x)+e^{-\rho \eta} h(x-z(\eta-s)) \frac{\partial \eta}{\partial s} \\
& +z \int_{s}^{\eta} e^{-\rho t} h_{x^{+}}(x-z(t-s)) d t-e^{-\rho \eta} h(x-z(\eta-s)) \frac{\partial \eta}{\partial s} \\
& +\int_{\eta}^{T} e^{-\rho t} h_{x^{+}}\left(x-z(\eta-s)+\left(\alpha_{2}-z\right)(t-\eta)\right)\left[z-z \frac{\partial \eta}{\partial s}-\alpha_{2} \frac{\partial \eta}{\partial s}\right] d t
\end{aligned}
$$

Owing to the fact that $\left[z-z \frac{\partial \eta}{\partial s}-\alpha_{2} \frac{\partial \eta}{\partial s}\right]$ is independent of $t, \psi(\eta)=x-z(\eta-s)$, replacing $s$ by $\eta$ in $(3.9)$ yields

$$
\int_{\eta}^{T} e^{-\rho t} h_{x^{+}}\left(x-z(\eta-s)+\left(\alpha_{2}-z\right)(t-\eta)\right) d t\left[z-z \frac{\partial \eta}{\partial s}-\alpha_{2} \frac{\partial \eta}{\partial s}\right]=0
$$


Thus,

$$
J_{s}(s, x)=-e^{-\rho s} h(x)+z \int_{s}^{\eta} e^{-\rho t} h_{x^{+}}(x-z(t-s)) d t .
$$

Similarly,

$$
\begin{aligned}
J_{x}(s, x)= & e^{-\rho \eta} h(x-z(\eta-s)) \frac{\partial \eta}{\partial x}+\int_{s}^{\eta} e^{-\rho t} h_{x^{+}}(x-z(t-s)) d t \\
& -e^{-\rho \eta} h(x-z(\eta-s)) \frac{\partial \eta}{\partial x}+\int_{\eta}^{T} e^{-\rho t} h_{x^{+}}\left(x-z(\eta-s)+\left(\alpha_{2}-z\right)(t-\eta)\right) d t\left[1-\alpha_{2} \frac{\partial \eta}{\partial x}\right] \\
= & \int_{s}^{\eta} e^{-\rho t} h_{x^{+}}(x-z(t-s)) d t .
\end{aligned}
$$

It can be seen that

$$
J_{x}(s, x) \geq \int_{s}^{\eta} e^{-\rho t} h_{x^{+}}(\psi(s)) d t \geq 0
$$

Therefore,

$$
\begin{aligned}
& J_{s}(s, x)+\min _{0 \leq u \leq \alpha_{2}}(u-z) J_{x}(s, x)+e^{-\rho s} h(x) \\
= & J_{s}(s, x)-z J_{x}(s, x)+e^{-\rho s} h(x) \\
= & 0 .
\end{aligned}
$$

By (1), (2), and (3), it can be proved that $J(s, x)$ is continuously differentiable. Finally, $v_{x}\left(s, \psi(s), \alpha_{2}\right)=0$.

Proof of Lemma 3.4. We divide the proof into four steps.

(1) $\phi(T)=0$ and $0 \leq \phi(s)<\psi(s)$ for $s \in[0, T)$.

Since $H(T, x)=h(x)$ and 0 is the unique minimizer of $h(x), \phi(T)=0$.

If $\phi(s)<0$, then $h(\phi(s))>h(0)$ and $v\left(s, \phi(s), \alpha_{2}\right) \geq v\left(s, 0, \alpha_{2}\right)$. Thus $H(s, \phi(s))>H(s, 0)$. But this contradicts the definition of $\phi(s)$. Hence, $\phi(s) \geq 0$.

If $\phi(s) \geq \psi(s)$, then $H(s, \phi(s)) \geq H(s, \psi(s))$ because $h(\phi(s)) \geq h(\psi(s))$ and $v\left(s, \phi(s), \alpha_{2}\right) \geq$ $v\left(s, \psi(s), \alpha_{2}\right)$. This means that $\phi(s) \leq \psi(s)$. Thus, $\phi(s)=\psi(s)$. However, if we take $\beta>0$ and small enough, then, as $\beta \rightarrow 0$,

$$
\begin{aligned}
& \frac{H(s, \psi(s))-H(s, \psi(s)-\beta)}{\beta} \\
\rightarrow & h_{x^{-}}(\psi(s))+\lambda \int_{0}^{T-s} e^{-\rho t} h_{x^{-}}\left(\psi(s)+\left(\alpha_{2}-z\right) t\right) d t>0 \\
= & h_{x^{-}}(\psi(s))+\lambda \int_{0}^{T-s} e^{-\rho t} h_{x^{+}}\left(\psi(s)+\left(\alpha_{2}-z\right) t\right) d t>0 \\
= & h_{x^{-}}(\psi(s))>0
\end{aligned}
$$

because $\psi(s)>0$ for $s<T$. In the above, $\lambda$ is the same as in $\S 3$. Therefore,

$$
H(s, \phi(s))>H(s, \psi(s)-\beta) \text { for } \beta>0 \text { and small enough. }
$$


This contradicts $\phi(s)=\psi(s)$. Hence, $\phi(s)<\psi(s)$ for $s \in[0, T)$.

(2) $\phi(s)$ is a continuous function.

Suppose this were not true. Then there would exist $\phi_{1}(s)<\phi_{2}(s)$ such that

$$
\tilde{H}\left(s, \phi_{1}(s)\right)=\tilde{H}\left(s, \phi_{2}(s)\right)=\min _{x} \tilde{H}(s, x) .
$$

Let $I_{s}=\left[\phi_{1}(s), \phi_{2}(s)\right] \subset[0, \psi(s)]$. Then the convexity of $\tilde{H}(s, x)$ yields that $\tilde{H}(s, x)=$ constant on $I_{s}$. Consequently, $\tilde{H}_{x}+(s, x)=0$ on $I_{s}$. Recall the definition of $\tilde{H}$ in (3.11) and (3.12). Meanwhile, notice that

$$
\tilde{H}_{x^{+}}(s, x)=h_{x^{+}}(x)+\int_{0}^{T-s} e^{-\rho t} h_{x^{+}}\left(x+\left(\alpha_{2}-z\right) t\right) d t=0
$$

and the fact that $h_{x^{+}}(x)$ is an increasing function. Thus,

$$
\begin{aligned}
& h_{x^{+}}(x)=\text { constant on } I_{s} \\
& \text { and } \quad \int_{0}^{T-s} e^{-\rho t} h_{x^{+}}\left(x+\left(\alpha_{2}-z\right) t\right) d t=\text { constant on } I_{s} .
\end{aligned}
$$

In particular,

$$
\int_{0}^{T-s} e^{-\rho t} h_{x^{+}}\left(\phi_{1}(s)+\left(\alpha_{2}-z\right) t\right) d t=\int_{0}^{T-s} e^{-\rho t} h_{x^{+}}\left(\phi_{2}(s)+\left(\alpha_{2}-z\right) t\right) d t .
$$

Because of the monotonicity of $h_{x^{+}}(x)$,

$$
h_{x^{+}}\left(\phi_{1}(s)+\left(\alpha_{2}-z\right) t\right)=h_{x^{+}}\left(\phi_{2}(s)+\left(\alpha_{2}-z\right) t\right) \text { for almost all } t \in[0, T-s] \text {. }
$$

Since $h_{x^{+}}(x)$ is an increasing function, $h_{x^{+}}(x)=$ constant for almost all $x \in\left(\phi_{2}(s)-\psi(s), \phi_{1}(s)\right)$. This implies that $h(x)$ is linear on $\left(\phi_{2}(s)-\psi(s), \phi_{1}(s)\right)$. By $(1)$, it is obvious that $0 \in\left(\phi_{2}(s)-\psi(s), \phi_{1}(s)\right)$. But this contradicts the fact that $h(x)>h(0)$ for all $x \neq 0$ in Assumption (A1). Finally, The continuity of $\phi(s)$ follows from the continuity of $\tilde{H}(s, x)$. This proves $(2)$.

(3) $\phi(s)$ is Lipschitz on $\left[0, T-\eta_{0}\right]$ for any $\eta_{0}>0$.

Let $s, s^{\prime} \in\left[0, T-\eta_{0}\right]$. Since $\phi(s)$ is continuous on $[0, T]$, it is uniformly continuous. Thus there exists a $\delta_{0}>0$ such that

$$
\left|\phi(s)-\phi\left(s^{\prime}\right)\right| \leq\left|\alpha_{2}-z\right| \eta_{0} / 2, \text { for all }\left|s-s^{\prime}\right| \leq \delta_{0}
$$

Let us consider those $\left|s-s^{\prime}\right| \leq \delta_{0}$ only. Without loss of generality, we may suppose that $\phi(s) \neq \phi\left(s^{\prime}\right)$, say $\phi\left(s^{\prime}\right)>\phi(s)$. Since $\phi(s)$ is the unique minimizer of $\tilde{H}(s, x)$ (by step $(2)$ ), for each $s$ and $n$ there exist $\phi_{1}^{n}(s)<\phi(s)<\phi_{2}^{n}(s)$ such that

$$
\begin{array}{ll} 
& \phi_{1}^{n}(s)<\phi(s)<\phi_{2}^{n}(s), \\
& \left|\phi_{1}^{n}(s)-\phi_{2}^{n}(s)\right| \leq 1 / n \\
\text { and } \quad & \tilde{H}\left(s, \phi_{1}^{n}(s)\right)=\tilde{H}\left(s, \phi_{2}^{n}(s)\right) .
\end{array}
$$


It follows that $\lim _{n \rightarrow \infty} \phi_{1}^{n}(s)=\lim _{n \rightarrow \infty} \phi_{2}^{n}(s)=\phi(s)$.

Let

$$
\begin{aligned}
I_{n} & =\frac{1}{s-s^{\prime}}\left[\frac{\tilde{H}\left(s, \phi_{1}^{n}(s)\right)-\tilde{H}\left(s, \phi_{2}^{n}(s)\right)}{\phi_{1}^{n}(s)-\phi_{2}^{n}(s)}-\frac{\tilde{H}\left(s^{\prime}, \phi_{1}^{n}(s)\right)-\tilde{H}\left(s^{\prime}, \phi_{2}^{n}(s)\right)}{\phi_{1}^{n}(s)-\phi_{2}^{n}(s)}\right] \\
\text { and } I_{n} & =\frac{1}{s-s^{\prime}}\left[\frac{\tilde{H}\left(s^{\prime}, \phi_{1}^{n}(s)\right)-\tilde{H}\left(s^{\prime}, \phi_{2}^{n}(s)\right)}{\phi_{1}^{n}(s)-\phi_{2}^{n}(s)}-\frac{\tilde{H}\left(s^{\prime}, \phi_{1}^{n}\left(s^{\prime}\right)\right)-\tilde{H}\left(s^{\prime}, \phi_{2}^{n}\left(s^{\prime}\right)\right)}{\phi_{1}^{n}\left(s^{\prime}\right)-\phi_{2}^{n}\left(s^{\prime}\right)}\right] .
\end{aligned}
$$

Then, $I_{n}+I I_{n}=0$.

We first show that there exists a constant $C$ such that $\left|I_{n}\right| \leq C$ for $n$ large enough. In fact,

$$
I_{n}=\frac{\lambda}{s-s^{\prime}} \int_{T-s^{\prime}}^{T-s} e^{-\rho t}\left[\frac{h\left(\phi_{1}^{n}(s)+\left(\alpha_{2}-z\right) t\right)-h\left(\phi_{2}^{n}(s)+\left(\alpha_{2}-z\right) t\right)}{\phi_{1}^{n}(s)-\phi_{2}^{n}(s)}\right] d t .
$$

Note that

$$
\left|\frac{h\left(\phi_{1}^{n}(s)+\left(\alpha_{2}-z\right) t\right)-h\left(\phi_{2}^{n}(s)+\left(\alpha_{2}-z\right) t\right)}{\phi_{1}^{n}(s)-\phi_{2}^{n}(s)}\right| \leq C_{1},
$$

where $C_{1}=\max \left\{\left|h_{x^{+}}\left(\left|\alpha_{2}-z\right| T+1\right)\right|,\left|h_{x^{-}}\left(-\left|\alpha_{2}-z\right| T-1\right)\right|\right\}<\infty$. Thus,

$$
\begin{aligned}
\left|I_{n}\right| & \leq\left|\frac{\lambda}{s-s^{\prime}} \int_{T-s^{\prime}}^{T-s} e^{-\rho t}\right| \frac{h\left(\phi_{1}^{n}(s)+\left(\alpha_{2}-z\right) t\right)-h\left(\phi_{2}^{n}(s)+\left(\alpha_{2}-z\right) t\right)}{\phi_{1}^{n}(s)-\phi_{2}^{n}(s)}|d t| \\
& \leq\left|\frac{\lambda C_{1}}{s-s^{\prime}} \int_{T-s^{\prime}}^{T-s} e^{-\rho t} d t\right| \\
& =\frac{\lambda C_{1}}{\rho}\left|\frac{e^{-\rho(T-s)}-e^{-\rho\left(T-s^{\prime}\right)}}{s-s^{\prime}}\right| \\
& \leq \frac{\lambda e^{-\rho T} C_{1}}{\rho} \sup _{s, s^{\prime}}\left|\frac{e^{\rho s}-e^{\rho s^{\prime}}}{s-s^{\prime}}\right| \\
& <\infty
\end{aligned}
$$

Let

$$
I I I_{n}=\frac{1}{\phi(s)-\phi\left(s^{\prime}\right)}\left[\frac{\tilde{H}\left(s^{\prime}, \phi_{1}^{n}(s)\right)-\tilde{H}\left(s^{\prime}, \phi_{2}^{n}(s)\right)}{\phi_{1}^{n}(s)-\phi_{2}^{n}(s)}-\frac{\tilde{H}\left(s^{\prime}, \phi_{1}^{n}\left(s^{\prime}\right)\right)-\tilde{H}\left(s^{\prime}, \phi_{2}^{n}\left(s^{\prime}\right)\right)}{\phi_{1}^{n}\left(s^{\prime}\right)-\phi_{2}^{n}\left(s^{\prime}\right)}\right] .
$$

Then

$$
\frac{\phi(s)-\phi\left(s^{\prime}\right)}{s-s^{\prime}}=\frac{I I_{n}}{I I I_{n}}
$$

Recall that $I_{n}+I I_{n}=0$. It follows that $\left|I_{n}\right|=\left|I_{n}\right| \leq C$. Thus to prove that $\phi(s)$ is Lipschitz on $\left[0, T-\eta_{0}\right]$, it suffices to show that there exists a constant $c>0$ such that $\left|I I I_{n}\right| \geq c$ for $n$ large enough. Let

$$
w(s, x)=e^{\rho s} v\left(s, x, \alpha_{2}\right)=\int_{0}^{T-s} e^{-\rho t} h\left(x+\left(\alpha_{2}-z\right) t\right) d t .
$$


Then, $\tilde{H}(s, x)=h(x)+\lambda w(s, x)$. We write $I I I_{n}=I I I_{n}^{\prime}+\lambda I I I_{n}^{\prime \prime}$ where

$$
I I I_{n}^{\prime}=\frac{1}{\phi(s)-\phi\left(s^{\prime}\right)}\left[\frac{h\left(\phi_{1}^{n}(s)\right)-h\left(\phi_{2}^{n}(s)\right)}{\phi_{1}^{n}(s)-\phi_{2}^{n}(s)}-\frac{h\left(\phi_{1}^{n}\left(s^{\prime}\right)\right)-h\left(\phi_{2}^{n}\left(s^{\prime}\right)\right)}{\phi_{1}^{n}\left(s^{\prime}\right)-\phi_{2}^{n}\left(s^{\prime}\right)}\right]
$$

and

$$
I I I_{n}^{\prime \prime}=\frac{1}{\phi(s)-\phi\left(s^{\prime}\right)}\left[\frac{w\left(s^{\prime}, \phi_{1}^{n}(s)\right)-w\left(s^{\prime}, \phi_{2}^{n}(s)\right)}{\phi_{1}^{n}(s)-\phi_{2}^{n}(s)}-\frac{w\left(s^{\prime}, \phi_{1}^{n}\left(s^{\prime}\right)\right)-w\left(s^{\prime}, \phi_{2}^{n}\left(s^{\prime}\right)\right)}{\phi_{1}^{n}\left(s^{\prime}\right)-\phi_{2}^{n}\left(s^{\prime}\right)}\right] .
$$

Observe that as $n \rightarrow \infty$,

$$
I I I_{n}^{\prime} \geq \frac{1}{\phi(s)-\phi\left(s^{\prime}\right)}\left[h_{x^{-}}(\phi(s))-h_{x^{+}}\left(\phi\left(s^{\prime}\right)\right)\right] \geq 0 .
$$

Furthermore,

$$
I I I_{n}^{\prime \prime} \rightarrow \frac{w_{x}\left(s^{\prime}, \phi(s)\right)-w_{x}\left(s^{\prime}, \phi\left(s^{\prime}\right)\right)}{\phi(s)-\phi\left(s^{\prime}\right)}, \text { as } n \rightarrow \infty
$$

If it can be shown

$$
\frac{w_{x}\left(s^{\prime}, \phi(s)\right)-w_{x}\left(s^{\prime}, \phi\left(s^{\prime}\right)\right)}{\phi(s)-\phi\left(s^{\prime}\right)} \geq c>0,
$$

then we are done. To this end, observe the following:

$$
\begin{aligned}
& \frac{w_{x}\left(s^{\prime}, \phi(s)\right)-w_{x}\left(s^{\prime}, \phi\left(s^{\prime}\right)\right)}{\phi(s)-\phi\left(s^{\prime}\right)} \\
= & \int_{0}^{T-s^{\prime}} e^{-\rho t}\left[\frac{h_{x^{+}}\left(\phi(s)+\left(\alpha_{2}-z\right) t\right)-h_{x^{+}}\left(\phi\left(s^{\prime}\right)+\left(\alpha_{2}-z\right) t\right)}{\phi(s)-\phi\left(s^{\prime}\right)}\right] d t \\
\geq & e^{-\rho T} \int_{0}^{T-s^{\prime}}\left[\frac{h_{x^{+}}\left(\phi(s)+\left(\alpha_{2}-z\right) t\right)-h_{x^{+}}\left(\phi\left(s^{\prime}\right)+\left(\alpha_{2}-z\right) t\right)}{\phi(s)-\phi\left(s^{\prime}\right)}\right] d t .
\end{aligned}
$$

The last inequality follows from the fact

$$
\frac{h_{x^{+}}\left(\phi(s)+\left(\alpha_{2}-z\right) t\right)-h_{x^{+}}\left(\phi\left(s^{\prime}\right)+\left(\alpha_{2}-z\right) t\right)}{\phi(s)-\phi\left(s^{\prime}\right)} \geq 0
$$

Since any convex function on $R^{1}$ is absolutely continuous, so is $h(x)$. Hence,

$$
\int_{a}^{b} h_{x^{+}}(x) d x=h(b)-h(a)
$$

for any $a$ and $b$. 
By (5.4),

$$
\begin{aligned}
& \frac{w_{x}\left(s^{\prime}, \phi(s)\right)-w_{x}\left(s^{\prime}, \phi\left(s^{\prime}\right)\right)}{\phi(s)-\phi\left(s^{\prime}\right)} \\
\geq & \left.\frac{e^{-\rho T}}{\phi(s)-\phi\left(s^{\prime}\right)}\left[-\frac{1}{\left|\alpha_{2}-z\right|} h\left(\phi(s)+\left(\alpha_{2}-z\right) t\right)+\frac{1}{\left|\alpha_{2}-z\right|} h\left(\phi\left(s^{\prime}\right)+\left(\alpha_{2}-z\right) t\right)\right]\right|_{0} ^{T-s^{\prime}} \\
= & \frac{e^{-\rho T}}{\phi(s)-\phi\left(s^{\prime}\right)}\left\{\left[\frac{1}{\left|\alpha_{2}-z\right|} h(\phi(s))-\frac{1}{\left|\alpha_{2}-z\right|} h\left(\phi\left(s^{\prime}\right)\right)\right]\right. \\
& \left.-\left[\frac{1}{\left|\alpha_{2}-z\right|} h\left(\phi(s)+\left(\alpha_{2}-z\right)\left(T-s^{\prime}\right)\right)-\frac{1}{\left|\alpha_{2}-z\right|} h\left(\phi\left(s^{\prime}\right)+\left(\alpha_{2}-z\right)\left(T-s^{\prime}\right)\right)\right]\right\} \\
= & \frac{e^{-\rho T}}{\left|\alpha_{2}-z\right|}\left\{\frac{h(\phi(s))-h\left(\phi\left(s^{\prime}\right)\right)}{\phi(s)-\phi\left(s^{\prime}\right)}\right. \\
& \left.-\frac{h\left(\phi(s)+\left(\alpha_{2}-z\right)\left(T-s^{\prime}\right)\right)-h\left(\phi\left(s^{\prime}\right)+\left(\alpha_{2}-z\right)\left(T-s^{\prime}\right)\right)}{\phi(s)-\phi\left(s^{\prime}\right)}\right\} \\
\geq & \frac{e^{-\rho T}}{\left|\alpha_{2}-z\right|}\left[h_{x}+(\phi(s))-h_{x}+\left(\phi\left(s^{\prime}\right)+\left(\alpha_{2}-z\right)\left(T-s^{\prime}\right)\right)\right] \\
\geq & \frac{e^{-\rho T}}{\left|\alpha_{2}-z\right|} c_{h}\left[\phi(s)-\phi\left(s^{\prime}\right)+\left|\alpha_{2}-z\right|\left(T-s^{\prime}\right)\right](\text { by Assumption }(\mathrm{A} 1) \\
\geq & \frac{e^{-\rho T}}{\left|\alpha_{2}-z\right|} c_{h}\left(-\frac{\left|\alpha_{2}-z\right| \eta_{0}}{2}+\left|\alpha_{2}-z\right|\left(T-s^{\prime}\right)\right)\left(\text { since } 0<\phi\left(s^{\prime}\right)-\phi(s) \leq \frac{\left|\alpha_{2}-z\right| \eta_{0}}{2}\right) \\
\geq & \frac{e^{-\rho T}}{\left|\alpha_{2}-z\right|} c_{h}\left(-\frac{\left|\alpha_{2}-z\right| \eta_{0}}{2}+\left|\alpha_{2}-z\right| \eta_{0}\right)\left(\text { since } T-s^{\prime} \geq \eta_{0}\right) .
\end{aligned}
$$

Hence,

$$
\frac{w_{x}\left(s^{\prime}, \phi(s)\right)-w_{x}\left(s^{\prime}, \phi\left(s^{\prime}\right)\right)}{\phi(s)-\phi\left(s^{\prime}\right)} \geq \frac{e^{-\rho T}}{2} c_{h} \eta_{0} .
$$

Therefore, for $n$ large enough,

$$
\left|\frac{\phi(s)-\phi\left(s^{\prime}\right)}{s-s^{\prime}}\right| \leq\left|\frac{I I_{n}}{I I I_{n}}\right| \leq \frac{C}{\eta_{0}}
$$

This implies that $\phi(s)$ is Lipschitz on $\left[0, T-\eta_{0}\right]$ for any $\eta_{0}>0$. Thus $\phi(s)$ is absolutely continuous on $\left[0, T-\eta_{0}\right]$. Consequently, $\phi(s)$ is differentiable a.e. on $\left[0, T-\eta_{0}\right]$. Since $\eta_{0}$ is arbitrary, $\phi(s)$ is differentiable on $[0, T]$ almost everywhere and $\int_{a}^{b} \dot{\phi}(s) d s=\phi(b)-\phi(a)$ for all $a, b \in[0, T)$. In the next step of the proof, we shall show that $0 \leq \dot{\phi}(s)+z \leq z$. This implies that $\dot{\phi}(s)$ is also bounded on $[0, T]$. Therefore, $\phi(s)$ is absolutely continuous on $[0, T]$, so for all $a, b \in[0, T]$, $\int_{a}^{b} \dot{\phi}(s) d s=\phi(b)-\phi(a)$.

(4) $\phi(s)$ is traceable.

Let $\rho(y)=\frac{1}{\sqrt{2 \pi}} \exp \left(-\frac{y^{2}}{2}\right)$ and $\rho_{\delta}(y)=\frac{1}{\delta} \rho\left(\frac{y}{\delta}\right)$. Then

$$
\int_{-\infty}^{\infty} \rho(y) d y=\int_{-\infty}^{\infty} \rho_{\delta}(y) d y=1
$$


Consider the convolution function $h^{\delta}$ of $\rho_{\delta}$ and $h$ defined by

$$
h^{\delta}(x)=\left(\rho_{\delta} * h\right)(x)=\int_{-\infty}^{\infty} \rho_{\delta}(y) h(x+y) d y \text {. }
$$

Then it follows that $h^{\delta} \in C^{\infty}\left(R^{1}\right)$ and, for all $N>0$,

$$
h^{\delta}(x)-h(x) \rightarrow 0 \text { uniformly on }|x| \leq N .
$$

Let $x^{\delta}$ denote the minimizer of $h^{\delta}(x)$. Then $x^{\delta} \rightarrow 0$ as $\delta \rightarrow 0$.

We first claim that $h^{\delta}(x)>h^{\delta}\left(x^{\delta}\right)$ for $x \neq x^{\delta}$. In fact, if this were not true, there would exist $x_{0}^{\delta}$, say $x_{0}^{\delta}<x^{\delta}$, such that $h^{\delta}\left(x_{0}^{\delta}\right)=h^{\delta}\left(x^{\delta}\right)$. This implies that $h_{x}^{\delta}(x)=0$ on $\left[x_{0}^{\delta}, x^{\delta}\right]$. Now, for any $x_{0}^{\delta} \leq x_{1} \leq x_{2} \leq x^{\delta}$, we have

$$
\int_{-\infty}^{\infty} \rho_{\delta}(y)\left[h_{x^{+}}\left(x_{2}+y\right)-h_{x^{+}}\left(x_{1}+y\right)\right] d y=0 .
$$

Since $h_{x^{+}}(x)$ is increasing in $x, h_{x^{+}}\left(x_{2}+y\right)-h_{x^{+}}\left(x_{1}+y\right)=0$ almost everywhere in $y \in(-\infty, \infty)$. This implies that $h(x)$ is linear in $(-\infty, \infty)$, which contradicts Assumption (A1). Thus, $h^{\delta}(x)>h^{\delta}\left(x^{\delta}\right)$ for $x \neq x^{\delta}$.

Define

$$
\tilde{H}^{\delta}(s, x)=h^{\delta}(x)+\lambda \int_{0}^{T-s} e^{-\rho t} h^{\delta}\left(x+\left(\alpha_{2}-z\right) t\right) d t .
$$

Let $\phi^{\delta}(s)$ denote the minimizer of $\tilde{H}^{\delta}(s, x)$, i.e.,

$$
\tilde{H}^{\delta}\left(s, \phi^{\delta}(s)\right)=\min _{x} \tilde{H}^{\delta}(s, x)
$$

Using similar arguments as before, it can be shown that $\phi^{\delta}(s)$ is a continuous curve such that $\phi^{\delta}(T)=0$ and $x^{\delta} \leq \phi^{\delta}(s)<x^{\delta}+\left|\alpha_{2}-z\right|(T-s)$ for $s \in(0, T)$. $\phi^{\delta}(s)$ is uniformly bounded and $\phi^{\delta}(s) \rightarrow \phi(s)$, as $\delta \rightarrow 0$.

To proceed, we claim that $\phi^{\delta}(s)$ is differentiable a.e. in $[0, T]$ for each $\delta>0$. Similarly as in proving the differentiability of $\phi(s)$, we only have to justify the inequality $(5.5)$ with $h^{\delta}(x)$ in place of $h(x)$ and with $s, s^{\prime} \in\left[0, T-\eta_{0}\right]$ and $0<\phi^{\delta}\left(s^{\prime}\right)-\phi^{\delta}(s) \leq\left|\alpha_{2}-z\right| \eta_{0} / 2$. It suffices to observe the 
following:

$$
\begin{aligned}
& \int_{0}^{T-s^{\prime}} \frac{h_{x}^{\delta}\left(\phi^{\delta}(s)+\left(\alpha_{2}-z\right) t\right)-h_{x}^{\delta}\left(\phi^{\delta}\left(s^{\prime}\right)+\left(\alpha_{2}-z\right) t\right)}{\phi^{\delta}(s)-\phi^{\delta}\left(s^{\prime}\right)} d t \\
= & \int_{0}^{T-s^{\prime}} \int_{-\infty}^{\infty} \rho_{\delta}(y)\left[\frac{h_{x}^{\delta}\left(\phi^{\delta}(s)+\left(\alpha_{2}-z\right) t+y\right)-h_{x}^{\delta}\left(\phi^{\delta}\left(s^{\prime}\right)+\left(\alpha_{2}-z\right) t+y\right)}{\phi^{\delta}(s)-\phi^{\delta}\left(s^{\prime}\right)}\right] d y d t \\
\geq & \int_{-x^{\delta}}^{\left|\alpha_{2}-z\right|\left(T-s^{\prime}\right)-x^{\delta}} \rho_{\delta}(y) \\
& \quad \times \int_{0}^{T-s^{\prime}}\left[\frac{h_{x}^{\delta}\left(\phi^{\delta}(s)+\left(\alpha_{2}-z\right) t+y\right)-h_{x}^{\delta}\left(\phi^{\delta}\left(s^{\prime}\right)+\left(\alpha_{2}-z\right) t+y\right)}{\phi^{\delta}(s)-\phi^{\delta}\left(s^{\prime}\right)}\right] d t d y \\
& (\operatorname{similar} \text { as in proving }(5.5)) \\
\geq & \int_{-x^{\delta}}^{\left|\alpha_{2}-z\right|\left(T-s^{\prime}\right)-x^{\delta}} \frac{\rho_{\delta}(y)}{\left|\alpha_{2}-z\right|}\left[h_{x}\left(\phi^{\delta}(s)+y\right)-h_{x^{+}}\left(\phi^{\delta}\left(s^{\prime}\right)+\left(\alpha_{2}-z\right)\left(T-s^{\prime}\right)+y\right)\right] d y \\
\geq & \int_{-x^{\delta}}^{\left|\alpha_{2}-z\right|\left(T-s^{\prime}\right)-x^{\delta}} \frac{\rho_{\delta}(y)}{\left|\alpha_{2}-z\right|}\left[c_{h}\left(\phi^{\delta}(s)-\phi^{\delta}\left(s^{\prime}\right)+\left|\alpha_{2}-z\right|\left(T-s^{\prime}\right)\right)\right] d y(\text { by Assumption (A1)) } \\
\geq & \frac{c_{h} \eta_{0}}{2\left|\alpha_{2}-z\right|} \int_{-x^{\delta}}^{\left|\alpha_{2}-z\right|\left(T-s^{\prime}\right)-x^{\delta}} \rho_{\delta}(y) d y \\
> & 0 .
\end{aligned}
$$

Hence, $\dot{\phi}^{\delta}(s)$ exists a.e. in $[0, T]$.

In what follows, it will be shown that

$$
0 \leq \dot{\phi}^{\delta}(s)+z \leq z
$$

Before proving (5.6), we first claim that (5.6) implies the following inequalities

$$
0 \leq \dot{\phi}(s)+z \leq z .
$$

In fact,

$$
\begin{aligned}
\frac{\phi(s)-\phi\left(s^{\prime}\right)+z\left(s-s^{\prime}\right)}{s-s^{\prime}} & =\lim _{\delta \rightarrow 0} \frac{\phi^{\delta}(s)-\phi^{\delta}\left(s^{\prime}\right)+z\left(s-s^{\prime}\right)}{s-s^{\prime}} \\
& =\lim _{\delta \rightarrow 0} \frac{1}{s-s^{\prime}} \int_{s}^{s^{\prime}}\left(\dot{\phi}^{\delta}(t)+z\right) d t .
\end{aligned}
$$

Since

$$
\dot{\phi}(s)+z=\lim _{s^{\prime} \rightarrow s} \frac{\phi(s)-\phi\left(s^{\prime}\right)+z\left(s-s^{\prime}\right)}{s-s^{\prime}}
$$

and

$$
0 \leq \frac{1}{s-s^{\prime}} \int_{s}^{s^{\prime}}\left(\dot{\phi}^{\delta}(t)+z\right) d t \leq z
$$

(5.7) holds.

To complete the proof of step (4), it suffices to justify (5.6). Observe that

$$
\begin{aligned}
0 & =\tilde{H}_{x}^{\delta}\left(s, \phi^{\delta}(s)\right) \\
& =h_{x}^{\delta}\left(\phi^{\delta}(s)\right)+\frac{\lambda}{\left|\alpha_{2}-z\right|} \exp \left(-\frac{\rho \phi^{\delta}(s)}{\left|\alpha_{2}-z\right|}\right) \int_{\phi^{\delta}(s)+\left(\alpha_{2}-z\right)(T-s)}^{\phi^{\delta}(s)} \exp \left(\frac{\rho t}{\left|\alpha_{2}-z\right|}\right) h_{x}^{\delta}(t) d t .
\end{aligned}
$$


Differentiating both sides of the above equation with respect to $s$,

$$
\begin{aligned}
0= & h_{x x}^{\delta}\left(\phi^{\delta}(s)\right) \dot{\phi}^{\delta}(s) \\
& +\frac{\lambda}{\left|\alpha_{2}-z\right|}\left(-\frac{\rho}{\left|\alpha_{2}-z\right|} \dot{\phi}^{\delta}(s)\right) \exp \left(-\frac{\rho \phi^{\delta}(s)}{\left|\alpha_{2}-z\right|}\right) \int_{\phi^{\delta}(s)+\left(\alpha_{2}-z\right)(T-s)}^{\phi^{\delta}(s)} \exp \left(\frac{\rho t}{\left|\alpha_{2}-z\right|}\right) h_{x}^{\delta}(t) d t \\
& +\frac{\lambda}{\left|\alpha_{2}-z\right|}\left[h_{x}^{\delta}\left(\phi^{\delta}(s)\right) \dot{\phi}^{\delta}(s)-\exp (-\rho(T-s)) h_{x}^{\delta}\left(\phi^{\delta}(s)+\left(\alpha_{2}-z\right)(T-s)\right)\left(\dot{\phi}^{\delta}(s)+\alpha_{2}-z \mid\right)\right] .
\end{aligned}
$$

Then we solve for $\dot{\phi}^{\delta}(s)$ to obtain

$$
\dot{\phi}^{\delta}(s)=\frac{F_{1}(\delta, s)}{F_{2}(\delta, s)}
$$

where

$$
\begin{aligned}
F_{1}(\delta, s)= & \lambda \exp (-\rho(T-s)) h_{x}^{\delta}\left(\phi^{\delta}(s)+\left(\alpha_{2}-z\right)(T-s)\right) \\
F_{2}(\delta, s)= & h_{x x}^{\delta}\left(\phi^{\delta}(s)\right)-\frac{\lambda \rho}{\left|\alpha_{2}-z\right|^{2}} \exp \left(-\frac{\rho \phi^{\delta}(s)}{\left|\alpha_{2}-z\right|}\right) \int_{\phi^{\delta}(s)+\left(\alpha_{2}-z\right)(T-s)}^{\phi^{\delta}(s)} \exp \left(\frac{\rho t}{\left|\alpha_{2}-z\right|}\right) h_{x}^{\delta}(t) d t \\
& +\frac{\lambda}{\left|\alpha_{2}-z\right|} h_{x}^{\delta}\left(\phi^{\delta}(s)\right)-\frac{\lambda}{\left|\alpha_{2}-z\right|} \exp (-\rho(T-s)) h_{x}^{\delta}\left(\phi^{\delta}(s)+\left(\alpha_{2}-z\right)(T-s)\right) .
\end{aligned}
$$

It is not difficult to see that:

$$
\begin{cases}h_{x}^{\delta}\left(\phi^{\delta}(s)+\left(\alpha_{2}-z\right)(T-s)\right) \leq 0 & \text { since } \phi^{\delta}(s) \leq x^{\delta}+\left|\alpha_{2}-z\right|(T-s) \\ h_{x}^{\delta}\left(\phi^{\delta}(s)\right) \geq 0 & \text { since } \phi^{\delta}(s) \geq x^{\delta} \\ h_{x x}^{\delta}\left(\phi^{\delta}(s)\right) \geq 0 & \text { since } h^{\delta}(x) \text { is convex. }\end{cases}
$$

Moreover, by (5.8),

$$
-\frac{\lambda \rho}{\left|\alpha_{2}-z\right|^{2}} \exp \left(-\frac{\rho \phi^{\delta}(s)}{\left|\alpha_{2}-z\right|}\right) \int_{\phi^{\delta}(s)+\left(\alpha_{2}-z\right)(T-s)}^{\phi^{\delta}(s)} \exp \left(\frac{\rho t}{\left|\alpha_{2}-z\right|}\right) h_{x}^{\delta}(t) d t=\frac{\rho}{\left|\alpha_{2}-z\right|} h_{x}^{\delta}\left(\phi^{\delta}(s)\right) \geq 0 .
$$

Thus, $F_{1}(\delta, s) \leq 0$ and $F_{2}(\delta, s) \geq 0$. This yields that $\dot{\phi}^{\delta}(s) \leq 0$. Hence, $\dot{\phi}^{\delta}(s)+z \leq z$. Note also that

$$
\begin{aligned}
& F_{1}(\delta, s)+\left|\alpha_{2}-z\right| F_{2}(\delta, s) \\
= & \left|\alpha_{2}-z\right| h_{x x}^{\delta}\left(\phi^{\delta}(s)\right)-\frac{\lambda \rho}{\left|\alpha_{2}-z\right|} \exp \left(-\frac{\rho \phi^{\delta}(s)}{\left|\alpha_{2}-z\right|}\right) \int_{\phi^{\delta}(s)+\left(\alpha_{2}-z\right)(T-s)}^{\phi^{\delta}(s)} \exp \left(\frac{\rho t}{\left|\alpha_{2}-z\right|}\right) h_{x}^{\delta}(t) d t \\
+ & \lambda h_{x}^{\delta}\left(\phi^{\delta}(s)\right) \geq 0 .
\end{aligned}
$$

Therefore,

$$
\dot{\phi}^{\delta}(s)+\left|\alpha_{2}-z\right|=\frac{F_{1}(\delta, s)+\left|\alpha_{2}-z\right| F_{2}(\delta, s)}{F_{2}(\delta, s)} \geq 0
$$

As a consequence,

$$
\dot{\phi}^{\delta}(s)+z \geq \alpha_{2} \geq 0 .
$$

The proof of Lemma 3.4 is completed. 


\section{Concluding remarks}

In this work, finite horizon optimal controls of stochastic manufacturing systems were considered. Time varying turnpike sets were obtained. Various properties of the underlying turnpike sets were analyzed. By utilizing the notion of traceability, existence of the optimal control policies was established and explicit formulas of the optimal controls were obtained via turnpike sets.

We would like to say a few words about the first problem considered in $\S 3$ in which repair facilities are available (or the strike can be resolved within that quarter). In this case, the machine capacity process $\alpha(t)$ is allowed to jump back and forth. The explicit optimal control of the resulting problem is very difficult to obtain. However, instead of searching for exact optimal solutions, one may be interested in finding approximate optimal solutions. If $\alpha(t)$ is assumed to be a finite state Markov chain (cf. [1] and [9]), then there exists a sequence of exponential random variables where the jumps of $\alpha(t)$ take place. One may write the value function $v$ as the limit of a sequence of value functions $v^{(n)}$ in which only $n$ jumps is allowed in the finite horizon under consideration (cf. [10]). In this way, the original stochastic control problem can be approximated by a sequence of deterministic control problems. For each $n$ one may also define curves $\phi(s)$ and $\psi(s)$ similarly as in sections 3 and 4 . The traceability of these curves are again difficult to verify analytically. One has to resort to numerical methods for solutions. Some previous discussions on numerical solutions for production planning problems can be found in [4], [8] and the references therein. Higher dimensional systems deserve further study and investigation.

We hope that this work will provide us with additional understanding in the finite horizon production planning for manufacturing systems. It is conceivable that the approach will render help and guidance on many practical applications.

\section{Acknowledgement}

The authors would like to thank Professor Ray Rishel for suggesting the problem and for helpful comments and discussions.

\section{References}

[1] R. Akella and P.R. Kumar, Optimal control of production rate in a failure-prone manufacturing system, IEEE Trans. Automat. Control AC-31 (1986), pp. 116-126. 
[2] A. Benveniste, M. Metivier and P. Priouret, Adaptive Algorithms and Stochastic Approximation, Springer-Verlag, Berlin, 1990.

[3] T. Bielecki and P.R. Kumar, Optimality of zero-inventory policies for unreliable manufacturing systems, Oper. Res. 26 (1988), pp. 532-546.

[4] K. Boukas, A. Haurie, and Ch. van Delft, A turnpike improvement algorithm for piecewise deterministic control, Optim. Control Appl. Meth. 12 (1991), 1-18.

[5] W.H. Fleming and R.W. Rishel, Deterministic and Stochastic Optimal Control. SpringerVerlag, New York, 1975.

[6] W.H. Fleming, S.P. Sethi and H.M. Soner, An optimal stochastic production planning problem with randomly fluctuating demand, SIAM J. Control Optim. 25 (1987), pp. 1494-1502.

[7] H. Ishii, Uniqueness of unbounded viscosity solution of Hamilton-Jacobi equations, Indiana Univ. Math. J. 33 (1984), pp. 721-748.

[8] J.G. Kimemia and S.B. Gershwin, An algorithm for the computer control production in flexible manufacturing systems, IIE Trans. 15 (1983), pp. 353-362.

[9] S.P. Sethi, H.M. Soner, Q. Zhang, and J. Jiang, Turnpike sets and their analysis in stochastic production planning problems, forthcoming in Math. Oper. Res. (1992).

[10] H.M. Soner, Optimal stochastic control with state-space constrains, II, SIAM J. Control Optim. 24 (1986), pp. 1110-1123. 